TRANSACTIONS OF THE

AMERICAN MATHEMATICAL SOCIETY

Volume 339, Number 2, October 1993

\title{
GEOMETRIC CURVATURE BOUNDS IN RIEMANNIAN MANIFOLDS WITH BOUNDARY
}

\author{
STEPHANIE B. ALEXANDER, I. DAVID BERG, AND RICHARD L. BISHOP
}

\begin{abstract}
An Alexandrov upper bound on curvature for a Riemannian manifold with boundary is proved to be the same as an upper bound on sectional curvature of interior sections and of sections of the boundary which bend away from the interior. As corollaries those same sectional curvatures are related to estimates for convexity and conjugate radii; the Hadamard-Cartan theorem and Yau's isoperimetric inequality for spaces with negative curvature are generalized.
\end{abstract}

\section{INTRODUCTION}

The main theorem of this paper characterizes Riemannian manifolds with boundary that have curvature bounded above in Alexandrov's sense by a preassigned constant. When the boundary is empty, this theorem corresponds to Alexandrov's basic theorem equating upper bounds on Alexandrov curvature to those on sectional curvature [ABN]. Thus Riemannian manifolds with boundary give rise to a large class of examples of metric spaces of curvature bounded above but not (since geodesics can bifurcate) bounded below. The characterization theorem leads to best possible global theorems on cut, conjugate and convexity radii in Riemannian manifolds with boundary. For example, the following intuitively appealing fact is a consequence of the characterization theorem and a theorem of Gromov on hyperbolic spaces: In a complete, simply connected, 3-dimensional space whose interior has nonpositive sectional curvature and whose boundary is everywhere saddle-shaped, any two points are joined by a unique geodesic.

The technique of proof of the characterization theorem is itself of interest. For example, it enables us to extend a linear isoperimetric inequality of Yau to manifolds with boundary.

A geodesic will be a locally distance-realizing curve parametrized proportionally to arclength; thus geodesics in manifolds with boundary can bend and bifurcate. A space has curvature bounded above by $K$, in the sense of Alexandrov, if every point has a neighborhood in which any minimizing geodesic triangle with vertices in the neighborhood has perimeter less than $2 \pi / \sqrt{K}$ (if $K>0$ ), and has each of its angles at most equal to the corresponding angle in a triangle with the same sidelengths in the standard surface $S_{K}$ of constant curvature

Received by the editors March 12, 1991 and, in revised form, July 3, 1991.

1991 Mathematics Subject Classification. Primary 53C20, 53B20, 53C45. 
$K$ [Av1]. Equivalently, as Alexandrov showed, the latter condition may be replaced by various others, for example, the "CAT*" condition that each distance between points on the triangle is at most equal to the distance in $S_{K}$ between the corresponding points on the comparison triangle, or the "CAT" condition that each distance from a vertex to a point on the opposite side is at most equal to the corresponding distance in $S_{K}$ ([Av1]; see also [Bn, Tv, ABN, Gv2]). Alexandrov worked in the general class of geodesic metric spaces, namely, those in which any two points are joined by a distance realizing curve, and used a generalized definition of angle. In this paper, we consider the subclass consisting of complete, connected Riemannian manifolds with boundary. Here angles in the Alexandrov sense coincide with Riemannian angles, and curvature bounded above by 0 is equivalent to local convexity, whereby every point has a neighborhood in which the distance $d(\gamma(t), \sigma(t))$ is convex for any two geodesics $\gamma$ and $\sigma$.

Characterization Theorem. Let $M$ be a Riemannian manifold with boundary $B$. Then the following two conditions are equivalent:

1. $M$ has curvature bounded above by $K$ in Alexandrov's sense.

2. The sectional curvatures of the interior of $M$ and the outward sectional curvatures of the boundary $B$ do not exceed $K$ (where an outward sectional curvature of $B$ is one that corresponds to a tangent section all of whose normal curvature vectors point outward).

This is a seemingly natural criterion, which was stated by Gromov for $K=0$ in [Gv1]. However, one should not expect this sharp bound for Alexandrov curvature to follow in any routine manner from the sectional curvature bounds. It is even a problem to establish that the Alexandrov curvature is locally bounded above. A weaker fact than this, namely that geodesics in manifolds with boundary are locally uniquely determined by their endpoints, was proved in [ABB1] and, independently, was the main theorem of [Si]. A characteristic difficulty, well known to workers in variational inequalities, lies in the possibility of unbounded switching behavior, which may, for example, produce Cantor coincidence sets between a geodesic and the boundary. Our approach, here and in [ABB1], has been to develop a geometric calculus of geodesic variations and Jacobi fields that works regardless of underlying pathology. One aim of this paper is to lay out these methods in a form convenient for others wishing to investigate Riemannian manifolds with boundary.

The characterization theorem places manifolds with boundary in the setting of Alexandrov's theory of spaces of curvature bounded above, and its recent extensions within Gromov's theory of hyperbolic groups. For example, by a theorem of Gromov, the Hadamard-Cartan theorem extends to geodesic metric spaces with curvature bounded above by 0 ([Gv1, Gv2]; see proof in [ArBp or $\mathrm{Bn}])$. Thus the characterization theorem immediately implies the following Hadamard-Cartan theorem for manifolds with boundary:

Corollary 1. If for a simply-connected, complete, connected Riemannian manifold with boundary, the sectional curvatures of the interior and the outward sectional curvatures of the boundary are nonpositive, then any two points are joined by a unique geodesic, and the distance between any two geodesics is convex.

For positive curvature bounds, we have a related result. Let $N(p, C)$ be the 
set of points, each of which can be joined to $p$ by a unique minimizing geodesic of length less than $C$. The characterization theorem and Alexandrov's comparison theory yield the following theorem on convexity of distance functions. We remark that a difficult direct proof of convexity preceded the characterization theorem, and is now obviated by it. Throughout, we set $1 / \sqrt{K}=\infty$ if $K \leq 0$.

Corollary 2. If for a complete Riemannian manifold with boundary, $M$, the sectional curvatures of the interior and the outward sectional curvatures of the boundary are no greater than $K$, then $N(p, \pi / 2 \sqrt{K})$ is open in $M$ and the distance function from $p$ is convex on $N(p, \pi / 2 \sqrt{K})$.

The characterization theorem will be proved by establishing the equivalence of conditions 1 and 2 with a third condition, namely, the $K$-convexity of normal Jacobi fields. This method leads to a proof of the following isoperimetric inequality, due to Yau in the case of Riemannian manifolds [Yu].

Theorem 2. If for a simply-connected, complete, connected, n-dimensional Riemannian manifold with boundary, $M$, the sectional curvatures of the interior and the outward sectional curvatures of the boundary are no greater than $K<0$, then the volume and perimeter of any measurable set $S$ in $M$ satisfy

$$
(n-1) \sqrt{-K} V(S) \leq P(S) .
$$

Theorem 2 does not seem to be accessible by standard arguments. Specifically, one might ask whether $M$ possesses an extension to a complete Riemannian manifold without boundary and with curvature no greater than $K+\varepsilon$, for arbitrary $\varepsilon$. While we do not know that this is false, we have verified that our assumptions do not allow this degree of curvature control among extensions obtained in the standard ways that suggest themselves, namely, by collaring the boundary and seeking either a conformal factor or a warping function depending on the distance from the boundary. For related computations, see [GIMr, pp. 196-198].

In what follows, $M$ always denotes a complete, $C^{\infty}$ Riemannian manifold with $C^{\infty}$ boundary $B$.

We thank M. Gromov for raising with us the question of proving the characterization theorem using the methods of [ABB1].

\section{JACOBI FIELDS}

A vector field $J$ along a geodesic $\gamma$ in $M$ will be called a Jacobi field if there is a sequence of geodesics $\gamma_{i}$ converging to $\gamma$ in the uniform topology, and a sequence of positive numbers $u_{i}$ approaching 0 for which

$$
\|J\|=\lim u_{i}^{-1} d\left(\gamma, \gamma_{i}\right)
$$

and the unit vector in the direction of $J(t)$ is the limit of the initial unit vectors of the minimizing geodesics from $\gamma(t)$ to $\gamma_{i}(t)$. (It makes no difference whether these distances and minimizers are taken in $M$, in some Riemannian extension $N$ of $M$ obtained by adding an outward collar along the boundary, or in some Euclidean space in which $N$ is isometrically immersed.) We say that the parametrized sequence $\left(\gamma_{i}, u_{i}\right)$ approaches $\gamma$ tangentially to $J$.

A Jacobi field $J$ along $\gamma$ is $K$-convex it if satisfies the differential inequality $\|J\|^{\prime \prime} \geq-K v^{2}\|J\|$, where $v$ is the speed of $\gamma$, in the following sense. We 
interpret the inequality to mean, if $K>0$, that on any parameter subinterval of length less than $\pi / v \sqrt{K}$, the sinusoid $a \sin (\sqrt{K} v t-b)$ that coincides with $\|J\|$ at the endpoints is an upper bound for $\|J\|$. If $K \leq 0$, we use the appropriate linear function or hyperbolic sinusoid instead, with no bound on the subinterval. In brief, the length of $J$ is bounded above, on appropriate subintervals, by the length of a normal Jacobi field in $S_{K}$ having the same endpoint lengths.

We begin by summarizing the existence, regularity and uniqueness properties of geodesics and Jacobi fields in $M$; except where otherwise noted, proofs may be found in [ABB1].

Geodesic properties. Existence. Any two points of $M$ are joined by a minimizing geodesic [C-V].

Regularity. A geodesic is $C^{1}$ and has one-sided acceleration everywhere. It possesses nonvanishing two-sided acceleration only on open segments which lie on the boundary $B$; this acceleration is necessarily outwardly normal to $B$. The acceleration fails to exist only at the countably many points where the geodesic switches from being a boundary geodesic with nonvanishing acceleration to being a geodesic in the interior or vice versa. In particular, at an accumulation point of such points, the acceleration exists and vanishes.

Uriqueness theorem. Each point of $M$ possesses a neighborhood of bipoint uniqueness $U$, any two of whose points are joined by a unique minimizing geodesic in $M$ and by no other geodesic in $U$.

Convergence theorem. If a sequence of geodesics converges pointwise, then the limit is a geodesic and the convergence is $C^{1}$.

Jacobi field properties. Existence theorem. If a parametrized sequence of geodesics $\left(\gamma_{i}, u_{i}\right)$ approaches a geodesic $\gamma$ tangentially to well-defined vectors at either endpoint, and if no subsequence approaches with infinite speed at any intermediate point (i.e., if $d\left(\gamma(t), \gamma_{i}(t)\right) \leq C u_{i}$ ), then some subsequence approaches $\gamma$ tangentially to a Jacobi field.

Regularity theorem. A Jacobi field $J$ is $k$-convex for some sufficiently large positive constant $k$. Moreover, $J$ is continuous on the interior of its interval of definition and $\|J\|$ is upper semicontinuous at the endpoints. It follows from $k$-convexity and continuity that $\|J\|$ has the regularity properties of a convex function on the interior of its domain. In particular, $\|J\|$ has left and right derivatives everywhere; there are only countably many points where $\|J\|^{\prime}$ fails to exist and at these points $\|J\|^{\prime}$ has a positive jump; and $\|J\|^{\prime \prime}$ exists almost everywhere.

Uniqueness. The existence and continuity of Jacobi fields are nontrivial, and we do not not know a corresponding uniqueness theorem; it is possible, for all we know, that different subsequences may produce more than one Jacobi field attached to a given one-sided variation. However, it turns out that uniqueness is immaterial to our arguments.

The purpose of this section is to prove the most difficult part of the characterization theorem, namely, that for normal Jacobi fields the convexity constant can be improved to $K$ if all interior and outward boundary sectional curvatures are at most $K$. (The restriction to normal Jacobi fields is unnecessary when $K \geq 0$, since then the inclusion of a tangential summand, which is linear in arclength (see $\S 6$ ), will only strengthen the $K$-convexity inequality.) 
Proposition 1. The sectional curvatures of the interior and the outward sectional curvatures of the boundary are at most $K$ if and only if all normal Jacobi fields are $K$-convex.

Proof. The essential step is, assuming the given sectional curvature bounds, to verify $K$-convexity at points where $\gamma$ touches the boundary and has vanishing acceleration. Indeed, where $\gamma$ lies in the interior of $M, J$ is a Jacobi field of the interior, and so $K$-convexity follows from the formula [BpCn, p. 177],

$$
\|J\|^{\prime \prime}(0)=\left[-K(J) A\left(J, \gamma^{\prime}\right)^{2}\|J\|^{-1}+A\left(J^{\prime}, J\right)^{2}\|J\|^{-3}\right](0) .
$$

Here, $A(x, y)$ denotes the area of the parallelogram spanned by $x$ and $y$. Where a relatively open subsegment of $\gamma$ lies on the boundary and has nowhere vanishing acceleration, $J$ coincides with a Jacobi field of $B$, except possibly at an endpoint of $\gamma$; this is because geodesics of $M$ sufficiently close to $\gamma$ contain segments lying on $B$ arbitrarily close to the given segment. Thus $K$ convexity follows from (2) and the fact that, by the curvature hypotheses and the Gauss equation, the sectional curvature of $B$ is at most $K$ for every section containing $\gamma^{\prime}$. At a point where $\gamma$ switches between a boundary segment with nonvanishing acceleration and a segment in the interior, $\|J\|$ is $K$-convex on both sides and $\|J\|^{\prime}$ has a nonnegative jump, so $\|J\|$ is $K$-convex on an open interval. So we only need to consider the remaining points of $\gamma$, at all of which the acceleration exists and vanishes.

Our method is to extend $M$ to a Riemannian manifold $N$ by adding an outward collar along the boundary, and then to express the length of $J$ as a limit of difference quotients of displacements measured through $N$. This is allowable because distance in $M$ is linearly approximated by distance in $N$ (in fact, the difference is on the order of the cube of either one), so that (1) holds whether $d$ is distance in $M$ or in $N$. The displacement function $f$ measured through $N$ from $\gamma$ to a nearby geodesic is $C^{1}$ and has one-sided second derivatives. For any $\varepsilon>0$, we shall show that in some neighborhood of a point $p$ at which $\gamma$ lies on $B$ and has vanishing acceleration, the displacement $f$ satisfies, from left and right, the inequality $f^{\prime \prime} \geq-(K+\varepsilon) f$. Since $\|J\|$ is a limit of these displacements with scale factors, it follows that $p$ lies in an open segment on which $J$ is $(K+\varepsilon)$-convex. Thus $\gamma$ is the union of open segments on which $J$ is either $K$-convex or $(K+\varepsilon)$-convex. But then, since $\varepsilon$ is arbitrary, $\|J\|$ must be $K$-convex. (This is a somewhat delicate procedure since examples show that $f^{\prime \prime} \geq-K f$ may fail to hold for all of the finite displacements $f$ in $N$.)

Let us specify the setting to which the problem has been reduced. We are given $\varepsilon>0$ and a unitspeed geodesic $\gamma$ of $M$ such that $\gamma^{\prime}(0)$ is tangent to $B$ and $\gamma^{\prime \prime}(0)=0$. We wish to show that any geodesic $\sigma$ of $M$ whose velocity vectors are sufficiently close to $\gamma^{\prime}(0)$ and whose displacement vectors through $N$ from $\gamma$ are sufficiently close to normal to $\gamma$ satisfies $f^{\prime \prime} \geq-(K+\varepsilon) f$, where $f(t)=d_{N}(\gamma(t), \sigma(t))$.

The displacement $f(t)$ is the length of the minimizing geodesic in $N$ from $\gamma(t)$ to $\sigma(t)$; for each $t$, we parametrize this geodesic by $u \in[0,1]$ with constant speed. Let $V(t, u)$ be the unit vector in the $u$-direction. Then $f^{\prime}$ depends only on the inner products of $V$ with the end velocities, by the first variation formula in $N$,

$$
f^{\prime}(t)=\left\langle\sigma^{\prime}(t), V(t, 1)\right\rangle-\left\langle\gamma^{\prime}(t), V(t, 0)\right\rangle .
$$


Since $\gamma$ and $\sigma$ are $C^{1}$, then $V(t, 1)$ and $V(t, 0)$ have continuous covariant $t$-derivatives, $V^{\prime}(t, 1)$ and $V^{\prime}(t, 0)$. Since $\gamma$ and $\sigma$ have left and right accelerations everywhere, the Liebnitz rule gives the left and right derivatives of $f$, both of which we denote by $f^{\prime \prime}$,

$$
\begin{aligned}
f^{\prime \prime}(t)= & \left\langle\sigma^{\prime \prime}(t), V(t, 1)\right\rangle-\left\langle\gamma^{\prime \prime}(t), V(t, 0)\right\rangle \\
& +\left\langle\sigma^{\prime}(t), V^{\prime}(t, 1)\right\rangle-\left\langle\gamma^{\prime}(t), V^{\prime}(t, 0)\right\rangle .
\end{aligned}
$$

For a fixed $t$, the expression $\left\langle\sigma^{\prime}(t), V^{\prime}(t, 1)\right\rangle-\left\langle\gamma^{\prime}(t), V^{\prime}(t, 0)\right\rangle$ has nothing to do with the manifold with boundary $M$; it would remain the same if $\gamma$ and $\sigma$ were replaced by geodesics $\bar{\gamma}$ and $\bar{\sigma}$ of $N$ having the same velocities at $t$. Letting $\bar{f}$ denote the displacement in $N$ between $\bar{\gamma}$ and $\bar{\sigma}$, we thus have, for this fixed value of $t$,

$$
\bar{f}^{\prime \prime}(t)=\left\langle\sigma^{\prime}(t), V^{\prime}(t, 1)\right\rangle-\left\langle\gamma^{\prime}(t), V^{\prime}(t, 0)\right\rangle .
$$

The second variation formula in $N$ implies

$$
\bar{f}^{\prime \prime}(t) \geq-\bar{f}(t) \int_{0}^{1}\left\|T^{\perp}\right\|^{2} K(T, V) d u,
$$

where $T$ is the Jacobi field of $N$ whose endpoint values are $\gamma^{\prime}(t)$ and $\sigma^{\prime}(t)$, $T^{\perp}$ is its normal component and $K(T, V)$ is the corresponding sectional curvature of $N$. We may arrange to work in a neighborhood in $N$ of $\gamma(0)$ so small that the sectional curvatures there are no greater than $K+\varepsilon_{1}$, for any given positive $\varepsilon_{1}$. Thus

$$
\bar{f}^{\prime \prime}(t) \geq-\left(K+\varepsilon_{1}\right) A \bar{f}(t)
$$

where $A$ is a bound for $\left\|T^{\perp}\right\|^{2}$ (an upper bound if $K$ is nonnegative and a lower bound if $K+\varepsilon$ is taken to be negative). For any given positive $\varepsilon_{2}$, if $\sigma^{\prime}(t)$ and $\gamma^{\prime}(t)$ are sufficiently close to $\gamma^{\prime}(0)$ and the displacement vector from $\gamma(t)$ to $\sigma(t)$ is sufficiently close to normal to $\gamma$, it follows that

$$
\bar{f}^{\prime \prime}(t) \geq-\left(K+\varepsilon_{2}\right) \bar{f}(t)
$$

Therefore, the above conditions guarantee, for all $t$,

$$
\left\langle\sigma^{\prime}(t), V^{\prime}(t, 1)\right\rangle-\left\langle\gamma^{\prime}(t), V^{\prime}(t, 0)\right\rangle \geq-\left(K+\varepsilon_{2}\right) f(t) .
$$

To control the remaining terms of $(3)$, namely $\left\langle\sigma^{\prime \prime}(t), V(t, 1)\right\rangle$ and $\left\langle\gamma^{\prime \prime}(t)\right.$, $V(t, 0)\rangle$, set $\sigma^{\prime \prime}=g Z_{\sigma}$ and $\gamma^{\prime \prime}=h Z_{\gamma}$, where $Z$ is the outward unit normal to the boundary. Here $g$ and $h$ vanish at nonboundary points, and at boundary points are nonnegative normal curvatures of $B$. Since $h(0)=0$ by assumption, and since normal curvatures vary continuously with boundary tangent directions, $g$ and $h$ may be made arbitrarily small by choosing $\sigma^{\prime}(t)$ and $\gamma^{\prime}(t)$ sufficiently close to $\gamma^{\prime}(0)$. If $\gamma(t)$ and $\sigma(t)$ are both on $B$, the factors $\left\langle Z_{\sigma}(t), V(t, 1)\right\rangle$ and $\left\langle Z_{\gamma}(t), V(t, 0)\right\rangle$ measure the amount by which the angle from $Z$ to $V$ deviates from a right angle, that is, the deviation of a secant line of $B$ away from the tangent planes at its endpoints. In a sufficiently small neighborhood of $\gamma(0)$, each of these factors is bounded in absolute value by $b f(t)$, that is, by the displacement $f$ times a constant $b>0$ which depends on the extrinsic curvature of $B$ in $N$. Thus we may ensure

$$
\left\langle\sigma^{\prime \prime}(t), V(t, 1)\right\rangle-\left\langle\gamma^{\prime \prime}(t), V(t, 0)\right\rangle \geq-\varepsilon_{3} f(t)
$$


for any $\varepsilon_{3}$. If only one of $\gamma(t)$ and $\sigma(t)$ is on $B$, the effect is to eliminate one of the left-hand terms in (5), while in the other term the angle between $Z$ and $V$ shifts in a way that is desirable. For instance, if $\sigma(t)$ is in the interior of $M$ while $\gamma(t)$ is on $B$, then at $\gamma(t)$ we have $\left\langle Z_{\gamma}(t), V(t, 0)\right\rangle \leq b f(t)$. Thus (5) holds in this case also. Combining (4) and (5) gives $f^{\prime \prime}(t) \geq-\left(K+\varepsilon_{2}+\varepsilon_{3}\right) f(t)$, thus completing the proof of $K$-convexity of normal Jacobi fields.

It is easy to prove the converse, namely that $K$-convexity implies the desired sectional curvature bounds. Indeed, consider a unitspeed geodesic $\gamma$ of $B$ for which $\gamma^{\prime}(0)$ lies in an outward plane section on which the second fundamental form of $B$ is definite. There is a variation of $\gamma$ through geodesics of $B$ such that the corresponding Jacobi field $J$ is normal to $\gamma$, has $J(0)$ nonvanishing and in the section, and has $J^{\prime}(0)$ vanishing. Near $\gamma(0)$, the definiteness condition implies that this variation consists of geodesics of $M$. Thus by assumption, $J$ is a $K$-convex Jacobi field of $M$. Then formula (2) provides the required link to the sectional curvature of $B$; the second term on the righthand side of (2) vanishes because $J^{\prime}(0)=0$. The same argument, applied to geodesic variations in the interior, shows that the sectional curvatures of the interior also are at most $K$. Finally, an outward section of $B$ on which the second fundamental form of $B$ is not definite has curvature at most $K$ by the Gauss equation.

\section{3. $K$-CONVEXITY AND AlEXANDROV CURVATURE BOUNDS}

Proposition 2. $M$ has curvature bounded above by $K$ in the sense of Alexandrov if and only if normal Jacobi fields are K-convex.

Proof. Assuming $K$-convexity, we shall obtain the upper curvature bound from a "development" argument of the kind introduced by Alexandrov [Av2], together with this lemma: Every point of $M$ has a neighborhood in which any geodesic variation whose endpoint curves are Lipschitz is itself Lipschitz. This Lemma is a consequence of Theorem 4 of [ABB1]. Thus about any given point, we may choose a neighborhood of bipoint uniqueness in $M$ such that for every minimizing triangle $\Delta p q r$ with vertices in the neighborhood, the variation map from $[0,1] \times[0,1]$ into $M$ determined by the minimizers from any vertex to the points of the opposite side is Lipschitz. We may also assume that each side lies in an open set on which the distance function from the opposite vertex is $C^{1}$. (See $\S 6$ for the first variation formula and the differentiability of distance in $M$.)

To verify the angle comparison condition for $\Delta p q r$, we develop the above variation onto a map from $[0,1] \times[0,1]$ into $S_{K}$ that also has the form of a cone over a curve. That is, to the vertex $p$ associate a point $\bar{p}$ in $S_{K}$. To the minimizing geodesic $\gamma_{u}$ which joins $p$ to a varying point of $q r$, associate a geodesic $\bar{\gamma}_{u}$ from $\bar{p}$ in $S_{K}$ of the same length $l(u)$, turning monotonically in $u$, in such a way that the two endpoint curves have the same speed for each value of $u$. This is easily seen to be possible because $l^{\prime}(u)$ coincides with the tangential component of the velocity of both endpoint curves, by the first variation formula for $M$. We thus obtain a comparison cone in $S_{K}$, two of whose sides, $\overline{p q}$ and $\overline{p r}$, are geodesics of the same length as $p q$ and $p r$ respectively and whose third side, $\overline{q r}$, is a curve of the same length as $q r$. 
Note that the angle $\bar{\theta}$ at $\bar{p}$ swept out by the comparison cone is less than $\pi$ since the lengths of the sides of the cone satisfy the strict triangle inequality. We shall use the Lipschitz property to show that the angle $\theta$ at $p$ in $\Delta p q r$ is no greater than $\bar{\theta}$. Since a model triangle in $S_{K}$ for $\Delta p q r$ may be obtained by replacing the curve $\overline{q r}$ in the comparison cone with a geodesic of the same length, thereby increasing the angle at $\bar{p}$, it will follow that the curvature of $M$ is bounded above by $K$.

In order to prove that $\theta$ is no greater than $\bar{\theta}$, consider two points, $x$ and $y$, different from $p$ and lying on $p q$ and $p r$ respectively. Choose $x$ and $y$ sufficiently close to $p$ that the shortest path $\bar{\tau}$ joining their images $\bar{x}$ and $\bar{y}$ in $S_{K}$ remains within the comparison cone. Then $\bar{\tau}$ intersects transversely each of the varying geodesics $\bar{\gamma}_{u}$, and satisfies a $C^{1}$ relation $t=t(u)$. It follows that the curve $\tau$ in $M$ given by $t=t(u)$ is Lipschitz in $M$. Therefore $\tau$ is differentiable almost everywhere and its length is obtained by integrating its speed. Now fix a value of $u$ at which $\tau$ is differentiable. There is at least one Jacobi field $J_{u}$ along $\gamma_{u}$ that arises from varying $u$ in the positive direction, by the existence theorem for Jacobi fields and the Lipschitz property of the variation. Moreover, the tangential component $T_{u}$ of $J_{u}$ is linear and the normal component $N_{u}$ is again a Jacobi field (see $\S 6$ ). Now let $\bar{J}_{u}$ be the Jacobi field in $S_{K}$ along $\bar{\gamma}_{u}$ determined by the comparison cone. Then $\bar{\tau}^{\prime}(u)$ decomposes into a tangential component of the same length as the tangential component of $\tau^{\prime}(u)$, and a normal component that coincides with the normal component $\bar{N}_{u}$ of $\bar{J}_{u}$. Since at $t=1$, the lengths $\left\|J_{u}\right\|$ and $\left\|\bar{J}_{u}\right\|$ coincide by construction, as do $\left\|T_{u}\right\|$ and $\left\|\bar{T}_{u}\right\|$, then so do $\left\|N_{u}\right\|$ and $\left\|\bar{N}_{u}\right\|$. Since $N_{u}$ is a normal Jacobi field, it is $K$-convex by assumption, which means that $\left\|N_{u}\right\|$ is no greater than $\left\|\bar{N}_{u}\right\|$ for all values of $t$. Therefore the speed at each point of differentiability of $\tau$ is no greater than the corresponding speed of $\bar{\tau}$, so the length of $\tau$ is not greater than that of the minimizer $\bar{\tau}$. We conclude that the distance between $x$ and $y$ is no greater than that between $\bar{x}$ and $\bar{y}$ for all $x$ and $y$ sufficiently close to $p$, and hence that $\theta$ is no greater than $\bar{\theta}$.

The converse argument, deriving the $K$-convexity of normal Jacobi fields from the upper curvature bound, is less delicate. Let $\gamma$ be a minimizing geodesic of length less than $\pi / \sqrt{K}$, parametrized by $[0,1]$ and lying in a model neighborhood of $M$, and $J$ be a normal Jacobi field along $\gamma$, corresponding to a sequence $\left(\gamma_{i}, u_{i}\right)$. The $K$-convexity of $J$ will follow from the first variation formula for $M$ and the CAT* distance comparison. Specifically, for the triangles $\Delta \gamma(0) \gamma(1) \gamma_{i}(1)$ and $\Delta \gamma_{i}(1) \gamma_{i}(0) \gamma(0)$, construct model triangles in $S_{K}$ that likewise are attached along and lie on opposite sides of their corresponding side. Thus we obtain a sequence of geodesic quadrilaterals in $S_{K}$, whose sides corresponding to $\gamma$ and $\gamma_{i}$ will be denoted by $\bar{\gamma}$ and $\bar{\gamma}_{i}$. By the first variation formula in $M$ and $S_{K}$, if $i$ is sufficiently large then the angle at $\bar{\gamma}(1)$ is arbitrarily close to $\pi / 2$ since the angle at $\gamma(1)$ is; and then so is the angle at $\bar{\gamma}(0)$. It follows that, for any $\varepsilon>0$, if $i$ is sufficiently large then the displacement in $S_{K}$ from $\bar{\gamma}$ to $\bar{\gamma}_{i}$ satisfies the $(K+\varepsilon)$-convexity inequality. Now consider the point at which $\bar{\gamma}(t) \bar{\gamma}_{i}(t)$ intersects the diagonal $\bar{\gamma}(0) \bar{\gamma}_{i}(1)$. The CAT* comparison applied to both image triangles implies that the distance in $M$ between $\gamma(t)$ and $\gamma_{i}(t)$ is no greater than that between their image points in $S_{K}$. Since the length of $J$ is the limit of a sequence of these distances with a scale factor, we see that $J$ is $(K+\varepsilon)$-convex for any positive $\varepsilon$, and hence is $K$-convex. 


\section{COROllaRies OF THE CHARACTERIZATION THEOREM}

The characterization theoremimmediately implies Corollary 1, the HadamardCartan-Gromov theorem for manifolds with boundary. We now consider the characterization theorem's further consequences. Throughout this section, all geodesics will be parametrized by $[0,1]$. We say $M$ has no conjugate points along a geodesic $\gamma$ from $p$ if the right-hand endpoint map on the space of geodesics from $p$, in the uniform metric, acts homeomorphically from a neighborhood of $\gamma$ onto a neighborhood of its right-hand endpoint [ArBp]. Then the characterization theorem and [ArBp, Theorem 3] imply (setting $\pi / \sqrt{K}=\infty$ if $K \leq 0)$ :

Corollary 3. If for a complete Riemannian manifold with boundary, $M$, the sectional curvatures of the interior and the outward sectional curvatures of the boundary are no greater than $K$, then $M$ has no conjugate points along geodesics of length less than $\pi / \sqrt{K}$.

In order to prove the isoperimetric inequality of the next section, we need an infinitesimal version of Corollary 3 :

Corollary 4. If for a complete Riemannian manifold with boundary, $M$, the sectional curvatures of the interior and the outward sectional curvatures of the boundary are no greater than $K$, and if $\gamma:[0,1] \rightarrow M$ is a geodesic from $p$ of length less than $\pi / \sqrt{K}$, then any tangent vector $w$ at $\gamma(1)$ that does not point out of $M$ is the right-hand value of a Jacobi field of $M$ along $\gamma$ that vanishes at $p$.

Proof. Choose a sequence of points that approaches $\gamma(1)$ tangentially to $w$ with respect to a parameter sequence $u_{i}$. Since the endpoint map is a local homeomorphism, there is a sequence of geodesics $\gamma_{i}$ having these points as right-hand endpoints and converging to $\gamma$. By the existence theorem for Jacobi fields, it suffices to show $d\left(\gamma(t), \gamma_{i}(t)\right) \leq C u_{i}$ for some constant $C$.

For this we use a global version of the CAT* comparison, valid in any Alexandrov space of curvature no greater than $K$. Specifically, for any (not necessarily minimizing) geodesic $\gamma$ from $p$ of length less than $\pi / \sqrt{K}$, and any geodesic $\sigma$ from $p$ sufficiently close to $\gamma$ in the uniform topology, there exists a triangle in $S_{K}$ having the same sidelengths as that formed by $\gamma, \sigma$ and the minimizer between their right-hand endpoints; furthermore, the distance between $\gamma(t)$ and $\sigma(t)$ is no greater than the distance between the corresponding points in the comparison triangle. A proof of this fact is given in [ArBp] using Alexandrov's technique for building global comparisons from local ones [Av2]. Returning now to $M$, we can use the characterization theorem and the comparison just discussed to estimate the distance $d\left(\gamma(t), \gamma_{i}(t)\right)$ in terms of its value at $t=1$, and thus obtain the corollary.

Still in the setting of Corollaries 3 and 4 , let $N(p, C)$ denote the points of $M$ that can be joined to $p$ by a unique minimizing geodesic of length less than $C$. For $0<C \leq \pi / \sqrt{K}$, Corollary 3 and the fact that sequences of minimizers from $p$ in $N(p, C)$ have convergent subsequences imply that $N(p, C)$ is an 
open neighborhood of $p$ in $M$, in which the minimizing geodesics from $p$ vary continuously with their right-hand endpoints. In this setting, the Alexandrov theory $[\mathrm{Av} 2, \S 3.8]$ easily yields a global version of the CAT comparison. Namely, for any minimizing geodesic triangle with vertex $p$ and perimeter less than $2 \pi / \sqrt{K}$ in $N(p, \pi / \sqrt{K})$, the distance from $p$ to any point of the opposite side is at most equal to the distance between the corresponding points in the model triangle in $S_{K}$. Therefore the distance function from $p$ is convex on $N(p, \pi / 2 \sqrt{K})$; this is stated in the introduction as Corollary 2.

We note that a theorem of the Hadamard-Cartan type was also proved by F.E. Wolter for a class of simply-connected 2-dimensional manifolds with boundary $M[\mathrm{Wr}$. His hypothesis is that $M$ can be imbedded as a closed subset, with locally rectifiable boundary curves, of a complete 2-dimensional Riemannian manifold having empty boundary and no conjugate points, and his technique of proof is adapted to that case.

\section{THE ISOPERIMETRIC INEQUALITY}

Now we prove the extension of the Yau linear isoperimetric inequality to manifolds with boundary, stated in the introduction as Theorem 2.

Proof of Theorem 2. It suffices to prove the inequality for open sets $S$ with smooth boundary $\Gamma$ and compact closure; here we are using the formulation of the problem discussed in [BoZr, $\S \S 34$ and 14]. Set

$$
c_{n}(K, l)=\int_{[0, l]} \sinh ^{n-1} \sqrt{-K} t d t / \sinh ^{n-1} \sqrt{-K} l .
$$

This is the ratio of the volume in the standard $n$-dimensional space of curvature $K$ of the ball of radius $l$ to the area of its boundary; the ratio increases with $l$ to its supremum $1 /(n-1) \sqrt{-K}$.

Let $p$ be any point of $M$. By Corollary 1 , the geodesics of $M$ from $p$ vary uniquely and continuously with their right-hand endpoints, and the distance in $M$ between any two of them is convex. Therefore by taking the cone of geodesics from $p$ to $\Gamma$ we obtain a Lipschitz continuous map $\Phi$ of $\Gamma \times[0,1]$ into $M$. By Rademacher's theorem [Fr, 3.1.6], $\Phi$ is differentiable almost everywhere. The absolute value of the Jacobian of $\Phi$ at a point of differentiability $(q, t)$ may be written $l(q) D(q, t)$, where $l(q)$ is the length of the geodesic $\gamma_{q}$ from $p$ to $q$. Here $D(q, t)$ may be obtained by taking an orthonormal frame $J_{1, q}, \ldots, J_{n-1, q}$ which is tangent to $\Gamma$ at $q$ (and meets $\gamma_{q}$ at nonacute angles); extending this frame to Jacobi fields $J_{1}, \ldots, J_{n-1}$ along $\gamma_{q}$, each of which corresponds to a sequence of geodesics in the cone from $p$ to $\Gamma$; and taking the $(n-1)$-volume spanned by their normal components $N_{1}, \ldots, N_{n-1}$ at $\gamma_{q}(t)$. The Jacobi fields $J_{i}$ exist by Corollary 4 .

Since $N_{i}$ is also a Jacobi field $(\S 6)$, it is $K$-convex by Proposition 1 , which means by definition that

$$
\left\|N_{i}(t)\right\| /\left\|N_{i}(1)\right\| \leq \sinh (\sqrt{-K} l(q) t) / \sinh (\sqrt{-K} l(q)) .
$$

Now $J_{2, q}, \ldots, J_{n-1, q}$ can be taken normal to $\gamma_{q}$, and $J_{1, q}$ at angle $\theta(q)$ to $\gamma_{q}$. Therefore the total $n$-volume subtended by $\Gamma$ (counting multiplicities), 
namely [Fr, 3.2.3] the integral of $l(q) D(q, t)$ over $\Gamma \times[0,1]$, is at most

$$
\begin{gathered}
\int_{\Gamma} \int_{[0,1]} l(q) \sin \theta(q) \sinh ^{n-1}(\sqrt{-K} l(q) t) / \sinh ^{n-1}(\sqrt{-K} l(q)) d t d v(q) \\
\leq \int_{\Gamma} c_{n}(K, l(q)) d v(q) \leq S(\Gamma) /(n-1) \sqrt{-K}
\end{gathered}
$$

where $S(\Gamma)$ is the $(n-1)$-volume of $\Gamma$. Every point in $S$ lies on a geodesic from $p$ with a minimizing extension that either lies infinitely in the interior of $M$ or else intersects the boundary of $M$. Since in either case such an extension intersects $\Gamma$, the theorem follows.

Obviously, if $M$ is compact with diameter $2 R$, one obtains the stronger inequality $V(S) \leq c_{N}(K, R) P(S)$.

We conclude this section by turning to isoperimetric spanning surfaces in manifolds with boundary. Suppose the interior and outward boundary sectional curvatures of $M$ are at most $K$, and $\alpha$ is a rectifiable closed curve through $p$ in $M$. Suppose $\alpha$ has length $l<2 \pi / \sqrt{K}$ and lies in $N(p, \pi / \sqrt{K}$ ) (see Corollary 2). It follows from the characterization theorem and Alexandrov's theory of surface area in spaces of curvature bounded above [Av2, $\S 5]$ that the cone of minimizing geodesics from $p$ to $\alpha$ has area at most that of a disk in $S_{K}$ of circumference $l$. Here area is defined as a lower limit of areas of abstractly "inscribed" polyhedra composed of model triangles. (We remark here that the technique of Alexandrov's proof has been significantly extended by Reshetnyak [Rk].) Using Jacobi field comparisons as above, one can adapt Alexandrov's proof so as to derive the same statement for the Riemannian area. In particular, by Corollary 1 , if $M$ is simply connected and $K$ is nonpositive, the above statement holds for any rectifiable closed curve $\alpha$ in $M$.

\section{APPENDIX: THE FIRST VARIATION FORMULA}

Finally we derive the first variation formula for geodesics in a Riemannian manifold with boundary (Proposition 3); its consequences for distance functions, tangential and normal components of Jacobi fields, and the representation of angles were used in the preceding sections. Throughout, we assume that geodesics are parametrized by $[0,1]$.

Proposition 3. For any geodesic $\gamma$ of $M$ and any parametrized sequence $\left(\sigma_{i}, u_{i}\right)$ of geodesics converging to $\gamma$ tangentially to a Jacobi field $J$,

$$
\lim u_{i}^{-1}\left[l\left(\sigma_{i}\right)-l(\gamma)\right]=l(\gamma)^{-1}\left(\left\langle J(1), \gamma^{\prime}(1)\right\rangle-\left\langle J(0), \gamma^{\prime}(0)\right\rangle\right) .
$$

Proof. First let $\gamma$ and $\sigma$ be arbitrary minimizers in $M$. Consider minimizers joining the respective endpoints of $\gamma$ and $\sigma$ that have lengths $s$ and $t$, and form respective angles of $\theta$ and $\phi$ with $\gamma^{\prime}$, and $\bar{\theta}$ and $\bar{\phi}$ with $\sigma^{\prime}$ (see Figure 1). We shall show

$$
\begin{array}{r}
s \cos \bar{\theta}-t \cos \bar{\phi}+o(\max \{s, t\})<l(\sigma)-l(\gamma) \\
<-s \cos \theta+t \cos \phi+o(\max \{s, t\}) .
\end{array}
$$

To obtain (6) we approximate $\sigma$ as in the figure by a broken geodesic path of length

$$
f(s)+l(\gamma)-\sqrt{s}-\sqrt{t}+g(t) \geq l(\sigma)
$$




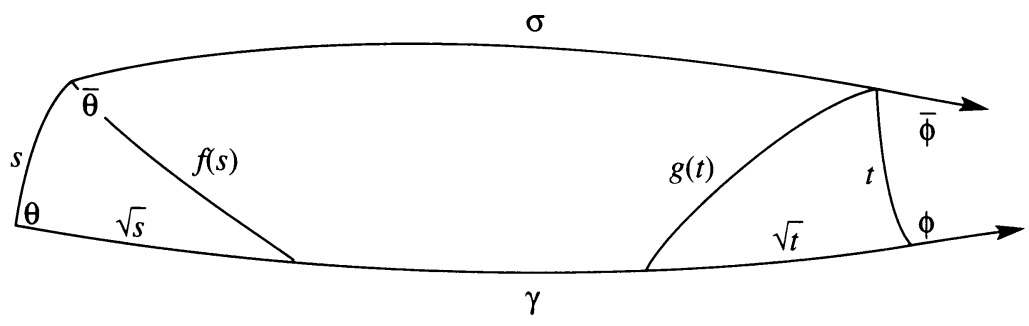

FIGURE 1

where $f(s)$ and $g(t)$ are the distances shown. Now consider the two end geodesic triangles, of sides $s, \sqrt{s}, f(s)$ and $t, \sqrt{t}, g(t)$ respectively. Using an isometric imbedding of $M$ in some Euclidean space $E$, we may also consider the Euclidean triangles having the same vertices. It is not hard to verify that the difference between distances measured in $M$ and in $E$ is on the order of the cube of either one. If these distances actually coincided, then the law of cosines would give

$$
s \cos \theta=\left(s^{2}+s-f(s)^{2}\right) / 2 \sqrt{s}=\sqrt{s}-f(s)+o(s),
$$

where the second equality is obtained by factoring $s-f(s)^{2}$ and noting that $|\sqrt{s}-f(s)| \leq s$ by the triangle inequality and hence that $f(s)=\sqrt{s}+o(\sqrt{s})$. A similar formula would hold for $t \cos \phi$. But the approximation implies that for the first triangle, each sidelength in $M$ differs from its Euclidean sidelength by $o(s)$ and for the second, by $o(t)$. Since the Euclidean angles approach the Riemannian ones, it follows from (7) that $l(\sigma)-l(\gamma)$ is at most $-s \cos \theta+$ $t \cos \phi+o(\max \{s, t\})$. Reversing the roles of $\gamma$ and $\sigma$ gives the remaining inequality in (6).

Now consider a parametrized sequence $\left(\sigma_{i}, u_{i}\right)$ of geodesics converging to $\gamma$ tangentially to $J$, and suppose first that the $\sigma_{i}$ and $\gamma$ are minimizers. Set $\sigma_{i}=\sigma$ above. Then for $i$ sufficiently large, $\theta, \bar{\theta}$ and $\phi, \bar{\phi}$ are approximately supplementary pairs by the convergence theorem for geodesics, that is, $\cos \theta+$ $\cos \bar{\theta}$ and $\cos \phi+\cos \bar{\phi}$ approach 0 . The desired formula follows immediately. Since every point has a neighborhood in which all geodesics are minimizers, and the formula is additive, the formula also holds for nonminimizing geodesics.

The formula (6) has the following immediate corollary, by holding left-hand endpoints fixed, so that $s=0$.

Corollary 5. The distance function $d_{p}$ from $p$ is differentiable except at those points $q$ that can be joined to $p$ by two minimizers that have different tangent directions at $q$; and $d_{p}$ is $C^{1}$ on the complement of the closure of the set of those points $q$.

F.-E. Wolter earlier proved the second statement by a different method [ $\mathrm{Wr}$, Theorem 3.2], in the course of his study of various definitions of cut locus in manifolds with boundary, and their relationships with each other and with the regularity properties of the distance function. The first statement is also useful in investigating the cut locus [ABB 2].

The following fact was used implicitly in $\S 3$, since the angle comparisons proved there were for Riemannian angles. 
Corollary 6. Riemannian angles in $M$ coincide with angles in Alexandrov's sense.

Proof. The Alexandrov angle at $p$ between two minimizers $\gamma_{1}$ and $\gamma_{2}$ is defined to be the limsup of all comparison angles in the Euclidean plane $S_{0}$ obtained by approaching $p$ along $\gamma_{1}$ and $\gamma_{2}$. That is, if points are chosen on $\gamma_{1}$ and $\gamma_{2}$ at distances $a$ and $b$ from $p$, respectively, and if $c$ is the distance between these points, then the cosine of the Alexandrov angle is

$$
\liminf _{a, b \rightarrow 0}\left(a^{2}+b^{2}-c^{2}\right) / 2 a b .
$$

We claim that in a manifold with boundary, this lim inf is a limit, and equals $\cos \theta$ where $\theta$ is the Riemannian angle between $\gamma_{1}$ and $\gamma_{2}$. To see this, it suffices to let $a$ and $b$ approach 0 along sequences for which the expression in (8) converges , $a \leq b$, and $a / b^{2}$ converges in $[0, \infty]$. If $a / b^{2}$ does not approach 0 , then the limit in (8) does not change if one substitutes distances measured through $E$ for $a, b$ and $c$, and so the claim is clear. If $a=o\left(b^{2}\right)$, then we may carry out the construction illustrated in Figure 1 with $s=a, t=0$, and $\gamma$ and $\sigma$ having sidelengths $b$ and $c$ respectively, to obtain

$$
a \cos \bar{\theta}+o(a)<c-b<-a \cos \theta+o(a)
$$

where $\theta$ is fixed as above. Using the fact that $\bar{\theta}$ approaches $\pi-\theta$, one shows that the expression in (8) approaches $\cos \theta$, as claimed.

Finally we note that using the first variation formula it is not hard to prove the following:

Corollary 7. The tangential component of a Jacobi field $J$ is linear. Its normal component is again a Jacobi field (except possibly if the base geodesic $\gamma$ meets $J$ in an acute angle at a boundary endpoint of $\gamma$ ).

\section{REFERENCES}

[Av1] A. D. Alexandrov, $A$ theorem on triangles in a metric space and some of its applications, Trudy Mat. Inst. Steklov. 38 (1951), 5-23. (Russian) (Much of [Av1] is translated in [Av2].)

[Av2] _ Über eine Verallgemeinerung der Riemannschen Geometrie, Schr. Forschungsinst. Math. 1 (1957), 33-84.

[ABN] A. D. Alexandrov, V. N. Berestovskii, and I. G. Nikolaev, Generalized Riemannian spaces, Russian Math. Surveys 41 (1986), 1-54.

[ABB1] S. B. Alexander, I. D. Berg, and R. L. Bishop, The Riemannian obstacle problem, Illinois J. Math. 31 (1987), 167-184.

[ABB2] __, Cut loci, minimizers and wave fronts in Riemannian manifolds with boundary, Michigan Math. J. 40 (1993) (to appear).

[ArBp] S. B. Alexander and R. L. Bishop, The Hadamard-Cartan theorem in locally convex spaces, Enseign. Math. 36 (1990), 309-320.

[Bn] W. Ballmann, Singular spaces of non-positive curvature. (E. Ghys and P. de la Harpe, eds.), Sur les Groupes Hyperboliques d'apres Mikhael Gromov, Birkhäuser, Boston, Basel, and Stuttgart, 1990.

[BpCn] R. L. Bishop and R. J. Crittenden, Geometry of manifolds, Academic Press, New York and London, 1964.

[BoZr] Yu. D. Burago and V. A. Zalgaller, Geometric inequalities, Springer-Verlag, Berlin, Heidelberg, and New York, 1988.

[C-V] S. Cohn-Vossen, Existenz Kurzester Wege, Doklady SSSR 8 (1935), 339-342. 
[Fr] H. Federer, Geometric measure theory, Springer-Verlag, Berlin, Heidelberg, and New York, 1969.

[GIMr] D. Gromoll and W. T. Meyer, Examples of complete manifolds with positive Ricci curvature, J. Differential Geom. 21 (1985), 195-211.

[Gv1] M. Gromov, Hyperbolic manifolds, groups and actions. (I. Kra and B. Maskit, eds.), Riemann Surfaces and Related Topics (Proc., Stony Brook, 1978), Ann. of Math. Studies, no. 97, Princeton Univ. Press, 1981, pp. 183-213.

[Gv2] — Hyperbolic groups. (S. M. Gersten, ed.), Essays in Group Theory, Math. Sci. Res. Inst. Publ., no. 8, Springer-Verlag, New York, Berlin, Heidelberg, 1987, pp. 75-264.

[Rk] Yu. G. Reshetnyak, Nonexpanding mappings in a space of curvature no greater than $K$, Sibirsk. Mat. Z. 9 (1968), 918-927; English transl. in Siberian Math. J. 9 (1968), 683-689.

[Si] D. Scollozi, Un risultato di locale unicita per le geodetiche su varieta con bordo, Boll. Un. Mat. Ital. B(6) 5 (1986), 309-327.

[Tv] M. Troyanov, Espaces a courbure negative et groupes hyperboliques. (E. Ghys and P. de la Harpe, eds.), Sur les Groupes Hyperboliques d'apres Mikhael Gromov, Birkhäuser, Boston, Basel, and Stuttgart, 1990.

[Wr] F.-E. Wolter, Cut loci in bordered and unbordered Riemannian manifolds, Technische Universität Berlin, FB Mathematik, Dissertation 249S, 1985.

[Yu] S.-T. Yau, Isoperimetric constants and the first eigenvalue of a compact Riemannian manifold, Ann. Sci. École. Norm. Sup. 8 (1975), 487-507.

Department of Mathematics, University of Illinois at Urbana Champaign, 273 Altgeld Hall, MC-382, 1409 West Green Street, Urbana, Illinois 61801

E-mail address: sba@symcom.math.uiuc.edu

E-mail address: berg@symcom.math.uiuc.edu

E-mail address: bishop@symcom.math.uiuc.edu 